\title{
DIFFERENTIABLE FUNCTIONS ON CERTAIN BANACH SPACES
}

\author{
BY ROBERT BONIC ${ }^{1}$ AND JOHN FRAMPTON \\ Communicated by G. A. Hunt, October 21, 1964
}

The main result in this note, Theorem 2 , can be thought of as a very strong maximum modulus type theorem. For example, let $D$ be a bounded connected open set in $C(0,1)$, and let $f: \mathrm{Cl} D \rightarrow R^{n}$ be continuous and differentiable in $D$. Then $f$ is determined by its values on the boundary of $D$. More exactly, $f(\mathrm{Cl} D) \subset \mathrm{Cl} f(\partial D)$. More generally, if $F$ is any Banach space and $f: \mathrm{Cl} D \rightarrow F$ is completely continuous and differentiable in $D$, then $f(\mathrm{Cl} D) \subset \mathrm{Cl} f(\partial D)$. Note that these results are false if $C(0,1)$ is replaced by a Hilbert space.

TheOREM 1. Let $D$ be a connected bounded open set in $l^{p}$ where $p$ is not an even integer. Assume $f$ is a real-valued function, continuous on $\mathrm{Cl} D$ and $n$-times differentiable in $D$ with $n \geqq p$. Then $f(\mathrm{Cl} D) \subset \mathrm{Cl} f(\partial D)$.

This generalizes a result proved in 1954 by Kurzweil [1]. Kurzweil assumed that $f$ was $n$-times continuously differentiable, that $D$ was a ball $B\left(x_{0}, r\right)$, and showed that inf $\left\{\left|f(x)-f\left(x_{0}\right)\right|:\left\|x-x_{0}\right\|=r\right\}=0$.

COROLlaRY 1. Let $f$ be an $n$-times differentiable function on $l^{p}$, where $n \geqq p$, and $p$ is not an even integer. If $f$ has its support in a bounded set, then $f$ is identically zero.

In particular, it follows that, for $n \geqq p, C^{n}$ partitions of unity do not exist whenever $p$ is not an even integer. This partially settles a question raised in Lang [2]. It should be noted, however, that this is implied by Kurzweil's result.

Corollary 2. Let $E$ be a Banach space containing a subspace equivalent to $l^{1}$. Assume $D$ is a connected bounded open set in $E$, and that $f$ is a real-valued function continuous on $\mathrm{Cl} D$ and differentiable in D. Then $f(\mathrm{Cl} D) \subset \mathrm{Cl} f(\partial D)$.

$C(0,1)$ and $L^{1}(0,1)$ are examples of spaces where Corollary 2 holds. More generally, any separable Banach space with an unconditional basis and nonseparable dual contains a subspace equivalent to $l^{1}$. It may be that any separable Banach space with a nonseparable dual has a subspace equivalent to $l^{1}$. Corollary 2 generalizes an unpublished result of Edward Nelson who showed that, in $C(0,1)$, differentiable functions with bounded support are identically zero.

\footnotetext{
${ }^{1}$ Research supported in part by NSF grant GP-1645.
} 
THEOREM 2. Let E be a Banach space containing a subspace equivalent to $l^{1}$, let $F$ be any Banach space, and let $D$ be a bounded open connected set in $E$. Assume $f: \mathrm{Cl} D \rightarrow F$ is continuous, and that $f^{\prime}(x)$ exists and is a completely continuous mapping for all $x \in D$. Then $f(\mathrm{Cl} D) \subset \mathrm{Cl} f(\partial D)$.

Corollary 1. Let $E$ and $F$ be as in the theorem and let $T: E \rightarrow F$ be completely continuous and differentiable. Then $T(\mathrm{Cl} D) \subset \mathrm{Cl} T(\partial D)$ for any bounded connected open set $D \subset E$.

This follows from the fact that if $T: E \rightarrow F$ is completely continuous and $T^{\prime}(x)$ exists, then $T^{\prime}(x)$ is a completely continuous linear mapping.

Letting $F$ be the reals gives the following "sup principle".

Corollary 2. Let $E$ and $D$ be as in the theorem, and let $f$ be a realvalued function continuous on $\mathrm{Cl} D$ and differentiable in $D$. Then $\sup _{C 1 D} f(x)=\sup _{\partial D} f(x)$.

Note that $f(x)=1-\|x\|^{2}$ shows that $E$ cannot be replaced by a Hilbert space.

Corollary 3. Let $M$ be a differentiable manifold modelled on $E$ where $E$ contains a subspace equivalent to $l^{1}$, and let $N$ be any differentiable manifold. Suppose $f: M \rightarrow N$ is differentiable and, for each $x$, $f^{\prime}(x): T_{x}(M) \rightarrow T_{f(x)}(N)$ is a completely continuous mapping. Let $(U, g)$ be a chart where $g U \subset E$ is bounded, open, and connected. Then $f(\mathrm{Cl} U)$ $\mathrm{CCl} f(\partial U)$.

It is well known that if $p$ is an even integer, the norm on $l^{p}$ is $C^{\infty}$, and if $p$ is not even the norm is $C^{q}$, where $q$ is the greatest integer strictly less than $p$. The argument in Lang [2] then shows that $C^{\infty}$ - and $C^{q}$-approximation holds in these respective spaces. It follows from Theorem 1 that for $p$ not even, $(q+1)$-differentiable approximation does not hold. Restrepo [3] showed that a Banach space has an equivalent $C^{1}$-norm if and only if its dual space is separable. It follows that $C^{1}$-approximation then holds for such spaces. It follows from Theorem 2 that if $E$ is a Banach space containing a subspace equivalent to $l^{1}$, then not even differentiable approximation holds. In the following we show that $C^{\infty}$-approximation holds for $c_{0}$. Restrepo's result shows that $c_{0}$ has an equivalent $C^{1}$-norm, and it is natural to ask if $c_{0}$ has an equivalent $C^{\infty}$-norm. However, we do not even know if $c_{0}$ has an equivalent $C^{2}$-norm. This result has also been observed by Edward Nelson. 
REMARK. $C^{\infty}$-approximation holds in $c_{0}$.

Simply let $g: R \rightarrow R$ be $a C^{\infty}$ function satisfying $g(t)=1$ for $|t| \leqq 1 / 2, g(t)=0$ for $|t| \geqq 1$, and $0<g(t) \leqq 1$ for $(1 / 2)<|t|<1$. Let $x=\left(x_{1}, x_{2}, \cdots\right) \in c_{0}$ and define $f(x)=\prod_{i-1}^{\infty} g\left(x_{i}\right)$. Then $f$ is a $C^{\infty}$ function nonzero in the open unit ball, and zero off it. The argument is then completed as in Lang [2].

Complete details, extensions, and applications of the results in this note will be published elsewhere.

\section{BIBLIOGRAPHY}

1. J. Kurzweil, On approximation in real Banach spaces, Studia Math. 14 (1954), 213-231.

2. S. Lang, Introduction to differentiable manifolds, Interscience, New York, 1962.

3. G. Restrepo, Differentiable norms in Banach spaces, Bull. Amer. Math. Soc. 70 (1964), 413-414.

Cornell UNIVERSITY AND

State University of New York at Stony Brook 